Scientific Electronic Archives

Issue ID: Sci. Elec. Arch. Vol. 15 (3)

March 2022

DOI: http://dx.doi.org/10.36560/15320221520

Article link: https://sea.ufr.edu.br/SEA/article/view/1520

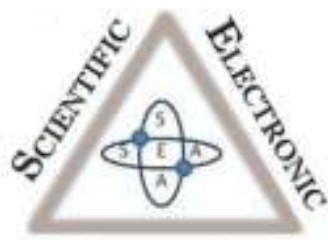

ARCHIVEs

ISSN 2316-9281

\title{
Agricultura de precisão: ferramenta de gestão na rentabilidade e produtividade de grãos
}

\section{Precision agriculture: management tool for profitability and grain productivity}

\author{
Lino Carlos Borges \\ Agência Goiana de Assistência Técnica, Extensão Rural e Pesquisa Agropecuária
}

\author{
Abadia dos Reis Nascimento \\ Universidade Federal de Goiás \\ Corresponding author \\ Cristiane Maria Ascari Morgado \\ Universidade Estadual de Goiás \\ cristiane.morgado@ueg.br
}

\begin{abstract}
Resumo. A agricultura de precisão é um processo gerencial. São várias as ferramentas utilizadas dentro da agricultura de precisão, tais como: eletrônica embarcada, geoestatística, sensores, etc, sendo que a tecnologia de informação é usada no manejo da propriedade e leva em consideração a variabilidade espacial e temporal que podem interferir na produtividade e rentabilidade. Nesse contexto, o objetivo deste trabalho foi realizar um levantamento bibliográfico, apresentando os conceitos de agricultura de precisão, seu foco na rentabilidade, bem como sua utilização na produção de grãos. Trata-se uma revisão bibliográfica simples em que foram pesquisados artigos científicos a respeito da agricultura de precisão e sua utilização com foco na rentabilidade e produção de grãos. Para tanto, as plataformas de pesquisa utilizadas foram o Google Acadêmico, o Scielo e a Elsevier, por meio das palavras-chave: agricultura de precisão, rentabilidade e produção de grãos. A agricultura de precisão na produção de grãos é uma ferramenta que auxilia o produtor na tomada de decisões, além de proporcionar economia, devido à redução da utilização de insumos e menor impacto no meio ambiente, se tornando, dessa forma, essencial para que informações sejam obtidas com o intuito de melhor entender e gerenciar os sistemas de produção agrícola. Além disso, a busca por mais informações e inovações por parte do agricultor brasileiro que o auxiliem na sua rotina de trabalho e aumentem sua rentabilidade faz com que a agricultura de precisão seja cada vez mais empregada, já que essa busca por inovações será essencial para destacar o Brasil quanto ao abastecimento mundial de alimentos nos próximos anos.
\end{abstract}

Palavras-chaves: tecnologias, GPS, SIG, processo gerencial.

\section{Abstract.}

Precision farming is a management process. There are several tools used in precision agriculture, such as: embedded electronics, geostatistics, sensors, etc. Information technology is used in property management and takes into account the spatial and temporal variability that can interfere with productivity and profitability. In this context, the objective of this work was to carry out a bibliographical survey, presenting the concepts of precision agriculture, its focus on profitability, as well as its use in grain production. This is a simple literature review in which scientific articles were searched about precision agriculture and its use with a focus on profitability and grain production. For this purpose, the research platforms used were Google Scholar, Scielo and Elsevier, using the keywords: precision agriculture, profitability and grain production. Precision agriculture in grain production is a tool that helps the producer in decision-making, in addition to providing savings, due to the reduction in the use of inputs and less impact on the environment, thus becoming essential for information to be in order to better understand and manage agricultural production systems. In addition, the search for more information and innovations by the Brazilian farmer to help them in their work routine and increase their profitability means that precision farming is increasingly used, as this search for innovations will be essential to highlight Brazil regarding the world food supply in the coming years.

Keywords: technologies, GPS, GIS, management process. 


\section{Contextualização e análise}

A agricultura teve seu início de forma pontual, em que "cada ponto da propriedade recebia somente os cuidados necessários, de forma localizada. Entretanto, isso era um entrave para as grandes propriedades, visto que este sistema de manejo era improdutivo e oneroso" (Fonseca, 2009, p.29). Ainda, a agricultura possui uma relação essencial entre a sociedade e o meio ambiente, fazendo com que a preocupação com os problemas ambientais bem como a dimensão da sustentabilidade se torne cada vez mais importantes nas pesquisas relacionadas ao agronegócio (Vitousek et al., 2009).

Outro ponto importante é que as propriedades agrícolas são desuniformes, tanto em relação aos fatores de produção, tais como: fertilidade, topografia, clima, quanto à produtividade obtida. Dessa forma, cada parte das plantações precisam de um manejo característico para a otimização da rentabilidade do produtor e minimizar os impactos ambientais (Pusch et al., 2019).

Assim, existiu a necessidade de se potencializar o manejo com o intuito de elevar a produtividade das grandes propriedades rurais. Assim, a busca por equipamentos tecnológicos se tornou cada vez maior. Além disso, o uso de uma estrutura potente e segura, a fim de diagnosticar os possíveis impactos ambientais, nas atividades do agronegócio, tem sido exposto como uma tendência em países líderes na produção de alimentos (Vitousek et al., 2009). Diante disso, essa maneira de realizar agricultura, considerando a desuniformidade das lavouras é chamada de Agricultura de Precisão.

Nos primórdios da agricultura era difícil o acesso a equipamentos tecnológicos suficientes para atender a demanda das grandes propriedades, então, a solução encontrada na época foi considerar a propriedade de forma homogênea, isto é, aplicar a mesma quantidade de insumos em toda a propriedade, sem considerar a variabilidade presente, proporcionando aumento da produtividade e fazendo com que os custos com o manejo se tornassem menos onerosos. Com o passar dos anos houve "o surgimento de novas tecnologias e isto fez com que os problemas quanto ao manejo localizado voltaram a rodear a agricultura, já que os equipamentos tecnológicos, que era o elo que faltava para permitir tal manejo, já existem" (Fonseca, 2009, p.29).

Além disso, o crescimento da população mundial deixa claro a pressão sobre os sistemas agrícolas e o aumento da demanda em converter as extensões remanescentes de ecossistemas naturais em agroecossistemas, o que fará com que os sistemas agrícolas se adequem para atender as necessidades de produção de alimentos (Foley et al., 2011).

Nesse contexto, soluções inovadoras são consideradas como o:
"Grande motor do desenvolvimento econômico e dos ganhos de produtividade e sustentabilidade. A capacidade de inovar é essencial para a obtenção e manutenção da competividade em um mercado global. Este fato é particularmente verdade no setor agropecuário, no qual novas tecnologias têm proporcionado aumentos significativos de produtividade com sustentabilidade" (Bassoi et al., 2019, p.19).

Os sistemas de produção agropecuários brasileiros vêm passando por modificações, as quais indicam a diminuição da mão de obra e, simultaneamente, para a intensificação do seu uso. Conforme IBGE (2015) mostrou que $84,72 \%$ da população brasileira era urbana e esse número tende a aumentar cada vez mais. Isto tem feito com que a população rural diminua, levando a um impacto na disponibilidade e qualidade da mão de obra no campo (Bassoi et al., 2019).

Manyika et al. (2017) relataram que $50 \%$ de todas as atividades hoje realizadas por trabalhadores poderão ser automatizadas até 2055 e que a revolução digital bem como a automação acontecerão tanto na agricultura quanto em setores de produção de alimentos. Diante disso, a utilização de automação, com máquinas, equipamentos e sensores será essencial para assegurar a segurança alimentar no futuro e tais mudanças serão importantes no sentido de trazer ganhos em eficiência, ajudando a agricultura a se tornar uma atividade cada vez mais sustentável (Bassoi et al., 2019). E é nesse contexto que a agricultura de precisão tem se tornado cada dia mais importante.

Várias aplicações referentes à utilização de GPS, equipamentos, dispositivos e programas computacionais direcionados à obtenção e processamento de dados georreferenciados vendo sendo relacionadas à prática da Agricultura de Precisão em lavouras de grãos no Brasil (Resende et al., 2014), e isto se torna cada vez mais importante já que a produção brasileira deve chegar a 333,1 milhões de toneladas nos próximos dez anos.

Entre as regiões produtoras, as que apresentaram maiores aumentos relativos tanto de produção quanto de área são Norte e Centro-Oeste, destacando-se os estados de Tocantins e Rondônia que devem liderar em produção e expansão, juntamente com o Mato Grosso, que segue na frente, liderando a produção de milho e soja no país (Brasil, 2021).

Diante do exposto, o objetivo deste trabalho foi realizar um levantamento bibliográfico, apresentando os conceitos de agricultura de precisão, seu foco na rentabilidade, bem como sua utilização na produção de grãos.

\section{Agricultura de precisão}

O termo agricultura de precisão se refere a uma prática agrícola na qual a tecnologia de informação é usada no manejo da propriedade e leva em consideração a variabilidade espacial e 
temporal que podem interferir na produtividade. Assim, levando-se em consideração esses fatores, pode-se obter maior retorno econômico ocasionar menor impacto ao meio ambiente (Mantovani, 2000; Caraski \& Mingotte, 2015). Outro aspecto da agricultura de precisão é que ela visa o "gerenciamento detalhado do sistema de produção agrícola como um todo, não somente nas aplicações de insumos ou de mapeamentos diversos, mas de todo os processos envolvidos na produção" (Cassman, 1999; Miranda, 2006).

Assim, a agricultura de precisão é um processo gerencial, que é iniciado a partir de informações exatas, concisas e se conclui com decisões exatas. É uma forma de administrar um campo produtivo metro a metro, considerando-se o fato de que cada área da propriedade possui características distintas (Roza, 2000).

Toledo et al. (2020) realizaram um levantamento bibliográfico sobre 0 uso da agricultura de precisão (geoestatística) no preparo de solo e concluíram que é preciso entender a variabilidade espaço-temporal das propriedades do solo para que se consiga o manejo correto da variabilidade espacial dos atributos químicos do solo, através das técnicas de geoestatística na avaliação.

O uso da agricultura de precisão no campo é considerado um processo seletivo, o qual não acontece de forma simultânea e contígua (Aracri, 2008). A definição de agricultura de precisão surgiu antes do momento da Revolução Industrial, como uma ferramenta para lidar com a cultura na procura do seu máximo de rendimento, considerando os fatores de localização, fertilidade do solo, entre outros. Os princípios que são conhecidos atualmente iniciaram-se no século $X X$, mas foi só na década de 80 na Europa e nos Estados Unidos, com 0 desenvolvimento dos computadores, sensores e softwares que ela se tornou viável para os produtores (Caraski \& Mingotte, 2015).

No ano de 1990 a agricultura de precisão chegou ao Brasil, sobretudo por meio das empresas multinacionais, e a tecnologia utilizada era totalmente importada. Esse início se deu através do levantamento da produtividade de grãos e com o georreferenciamento na agricultura. É uma ferramenta essencial no cenário agrícola brasileiro e o seu crescimento trará retornos positivos para a agricultura nacional, por meio da potencialização dos investimentos de recursos na produção (Molin, 2001).

A agricultura de precisão tem início na "coleta de dados, análises e interpretação dessas informações, bem como geração das recomendações, aplicação no campo e avaliação dos resultados" (Geebers \& Adamchuk, 2010). Assim, ela pode ser considerada uma sequência de conhecimentos, na qual máquinas, dispositivos, equipamentos e softwares são instrumentos para a coleta de dados, os quais precisam ser organizados e interpretados, suscitando informações para apoiar a gestão (Inamasu \& Bernardi, 2014).
Considerada como uma ferramenta que atua em três pontos da agricultura geral, assim, seu uso está ligado a solucionar problemas de ao menos um desses pontos: a produção, a administração da propriedade e o meio ambiente. Quanto à produção, o foco da agricultura de precisão $(A P)$ é melhorar o processo de produção e consequentemente, aumentar a eficiência da produção; quanto à administração da propriedade, a AP é adotada como ferramenta de gestão, ajudando na tomada e decisões. E por fim, quanto ao meio ambiente, seu uso pode reduzir os danos causados e levar a uma produção com responsabilidade ambiental (Fonseca, 2009).

Um dos pontos positivos da agricultura de precisão consiste no gerenciamento localizado dos talhões e a obtenção de um grande número de informações que auxiliarão para a tomada de decisões, o que significa ganhos não mensurados ou contabilizados até então. Devido a isso, a AP é considerada, antes de tudo, um sistema de gestão ou gerenciamento da produção agrícola (Balastreire, 2000).

A Committee que é composta pela Secretaria da Agricultura do governo americano (COMMITTEE 1997), considera a Agricultura de Precisão como uma ferramenta de gestão que usa as tecnologias da informação para aportar os dados de múltiplas fontes e auxiliar nas decisões pertinentes à produção vegetal, o que traz benefícios significativos para os produtores, que tem cada vez mais utilizado esta ferramenta.

Com base no exposto, tem-se que a agricultura de precisão envolve a utilização de tecnologias atualizadas para manejar o solo, insumos bem como as culturas e isso é feito de forma apropriada, considerando-se as variações espaciais e temporais nos quesitos que interferem a produtividade das mesmas. Além disso, o uso da AP está ligado ao uso das tecnologias como: sensoriamento remoto, sistemas de informações geográficas (SIG) e o sistema de posicionamento global (GPS). Assim, a AP é um sistema que envolve o desenvolvimento e o uso de tecnologias de gestão, baseando-se no objetivo principal de maximizar a rentabilidade, proporcionando a administração de cada parte do campo de forma adequada e se é um processo econômico e vantajoso fazer a administração dessa maneira (Dallmeyer \& Schlosser, 1999).

\section{Agricultura de precisão na produção de grãos}

Os diferentes princípios da agricultura de precisão vêm sendo usados com maior constância na produção de grãos, cana-de-açúcar, hortícolas, frutíferas e silvicultura, (Bernardi \& Inamasu, 2014), entretanto, é uma tecnologia que apresenta potencial elevado para ser usada em qualquer sistema agropecuário, inclusive em sistemas integrados.

A agricultura de precisão tem chamado a atenção do agricultor por estar associada à ideia de que as máquinas agrícolas que possuem acessórios 
modernos possuem maior autonomia e melhor desempenho operacional no campo, o que pode elevar o rendimento do trabalho na propriedade rural. Este fato tem feito com que as práticas agrícolas baseadas em georreferenciamento têm maior ocorrência entre os produtores de grãos em grande escala, já que os de porte pequeno ainda não almejam chances de desfrutar das vantagens desta ferramenta (Resende et al., 2014).

O uso da agricultura de precisão é mostrado como uma solução para aumentar a produção de alimentos, quando se compara a produtividade na cultura da soja por exemplo, a qual apresentou maiores médias em relação ao sistema convencional. Outro aspecto importante a ser considerado é que países como Brasil, Argentina e EUA poderiam obter incremento produtivo com 0 uso da agricultura de precisão, pelo fato de já terem alguma base tecnológica para produzir soja (Artuzo et al.,2017).

O uso das ferramentas oferecidas pela agricultura de precisão tem refletido na produção agrícola nacional, visto que o Brasil tem aumentado a produção de grãos como a soja por exemplo, o que pode ser verificado no relatório de safras elaborado pela Companhia Nacional de Abastecimento (Conab) no qual o Brasil produziu em torno de 119 milhões de toneladas de soja na safra 2018/2017 (CONAB, 2020).

As ferramentas da agricultura de precisão que apresentaram maior contribuição para a evolução dos patamares produtivos foram:

"o uso de instrumentos de navegação por satélite nas máquinas agrícolas, o mapeamento da variabilidade de fertilidade dos solos, a aplicação de fertilizantes e corretivos com taxas variáveis, monitoramento instantâneo de condições de planta, controle georreferenciado de pulverizações e a utilização de mapas de produtividade" (Santi et al., 2013, p. 511).

Ferraz et al. (2012) relataram que para a cultura do café, a agricultura de precisão é considerada como as técnicas e tecnologias que podem ajudar o produtor de café quanto ao manejo da sua lavoura, com base na variabilidade espacial tanto dos atributos do solo quanto da planta, com o objetivo de potencializar a rentabilidade, elevar a eficiência da adubação, bem como a pulverização e colheita, resultando no aumento da produtividade e a qualidade final dos grãos.

Silva et al. (2007) verificou a viabilidade econômica da utilização da agricultura de precisão na cultura de milho e soja no Mato Grosso do Sul, sendo usada como subsidio para tomada de decisões pelos produtores e concluíram que os índices de rentabilidade calculados mostraram melhores resultados econômicos no sistema de agricultura de precisão.

Soares Filho e Cunha (2015) realizaram um trabalho com o intuito de analisar o processo de adoção e de utilização das tecnologias de agricultura de precisão por produtores rurais do sudoeste do Estado de Goiás, visto que tal tecnologia apresenta um excelente campo para seu desenvolvimento. Através da aplicação de questionários aos produtores os autores concluíram, com base nos parâmetros avaliados, que a agricultura de precisão na região ainda estava em fase inicial de adoção e que a amostragem de solo em grade bem como a adubação a taxa variada a lanço foram as tecnologias mais utilizadas.

Preceitos da agricultura de precisão foram determinados por Dalchiavon et al. (2011) em condições de Cerrado (Selvíria, MS) analisando-se as correlações lineares e espaciais dos componentes de produção bem como a produtividade da soja com a resistência mecânica à penetração de um Latossolo, em sistema de semeadura direta. Com base os resultados obtidos, os autores constataram que os valores dos alcances da dependência espacial a serem utilizados devem estar entre $38,1 \mathrm{~m}$ e $114,7 \mathrm{~m}$, para trabalhos que vislumbrem a utilização dos mesmos atributos do seu estudo, voltados à agricultura de precisão.

Corassa et al. (2018) identificaram os atributos químicos do solo restritivos à produtividade de grãos em Latossolos sob sistema plantio direto, com uso de amostragem de solo dirigida e concluíram que quando as zonas de produtividade são consideradas na amostragem de solo, é uma maneira efetiva para se identificar os atributos químicos restritivos à produtividade de grãos, além de oferecer orientações quanto a intervenções sitioespecíficas mais precisas.

A definição de zonas de produtividade em áreas manejadas com agricultura de precisão foi estudada por Santi et al. (2011), onde os dados de produtividade foram obtidos por uma colhedora dotada de sistema Fieldstar ${ }^{\circledR}$ para a agricultura de precisão. Os resultados obtidos permitiram aos autores concluir que a sobreposição de ao menos três mapas de produtividade oferece a diferenciação de áreas com produtividades semelhantes ao longo dos anos, além disso, a filtragem de dados é crucial para a eliminação de erros e análise temporal dos mapas.

\section{Considerações finais}

A agricultura de precisão na produção de grãos é uma ferramenta que auxilia o produtor na tomada de decisões, além de proporcionar economia, devido à redução da utilização de insumos e menor impacto no meio ambiente, se tornando, dessa forma, essencial para que informações sejam obtidas com o intuito de melhor entender e gerenciar os sistemas de produção agrícola.

Além disso, a busca por mais informações e inovações por parte do agricultor brasileiro que o auxiliem na sua rotina de trabalho e aumentem sua rentabilidade faz com que a agricultura de precisão seja cada vez mais empregada, já que essa busca por inovações será essencial para destacar o Brasil quanto ao abastecimento mundial de alimentos nos próximos anos. 
Vale destacar que a agricultura de precisão é, antes de tudo, um processo gerencial e as ferramentas tecnologias como GPS, geoestatística, eletrônica embarcada, etc, são acessórios. A grande meta da agricultura de precisão é a rentabilidade. A produtividade pode ser alcançada, mas não é considerada o foco principal.

\section{Referências}

ARACRI, L. A. A difusão de inovações numa perspectiva crítica: práticas espaciais e a implantação da "agricultura de precisão" na microrregião de Canarana - MT. XV Encontro Nacional de Geógrafos. Anais... São Paulo: AGB, 2008.

ARTUZO, F. D.; FOGUESATTO, C. R.; SILVA, L. X. da. Agricultura de precisão: inovação para a produção mundial de alimentos e otimização de insumos agrícolas. Revista Tecnologia Sociedade, v. 13, n. 29, p. 146161,2017. DOI:

https://doi.org/10.3895/rts.v13n29.4755

BAIO, F.H.R. Aplicação de AP no Plantio. In: RIPOLI, T.C.C.; RIPOLI, M.L.C.; CASAGRANDI, D.V.; IDE, B.Y. (Org.). Plantio de cana-de-açúcar: estado da arte. 2.ed. Piracicaba: T.C.C. Ripoli, 2007, v. 1, p. 92-101.

BALASTREIRE, L. A. Potencial do uso da agricultura de precisão no Brasil. In: Encontro Paulista de Soja, 2, 2000. Campinas. Anais... Campinas, 2000. p. 176-217.

BASSOI, L.H.; INAMASU, R.Y. BERNARDI, A.C.C.; VAZ, C.M.P.; SPERANZA, E.A.; CRUVINEL, P.E. Agricultura de precisão e agricultura digital. In: TECCOGS - Revista Digital de Tecnologias Cognitivas, n. 20, p. 17-36, 2019. DOI: https://doi.org/10.23925/1984-3585.2019i20p17-36

BERNARDI, A. C.; INAMASU, R. Y. Adoção da agricultura de precisão no Brasil. In: BERNARDI, A. C. C.; NAIME, J. M.; RESENDE, A. V.; BASSOI, L. H.; INAMASU, R. Y. Agricultura de precisão: resultados de um novo olhar. Brasília, DF: Embrapa, 2014. p. 559-577.

BRASIL. Produção de grãos crescerá $27 \%$ nos próximos dez anos. Disponível em: <https://www.gov.br/ptbr/noticias/agricultura-e-pecuaria/2021/07/producao-degraos-crescera-27-nos-proximos-dez-anos>. Acesso em 20 out. 2021.

CARASKI, B.G.; MINGOTTE, F.L.C. Agricultura de precisão no cultivo de cana-de-açúcar. In: III SIMTEC Simpósio de Tecnologia da FATEC Taquaritinga. Disponível em: <www.fatectq.edu.br/simtec> 10 p. Outubro de 2015.

CORASSA, G. M.; SANTI, A. L.; SILVA, V. R.; BARON, F. A.; REIMCHE, G. B.; FIORESI, D.; FLORA, D. P. D. Soil Chemical atributes restricting grain yield in Oxisol sunder no-tillage system. Pesquisa Agropecuária Brasileira, v. 53, n. $11, \quad$ p. 1203-1212, 2018. DOI: https://doi.org/10.1590/S0100-204X2018001100002

CASSMAN, K. G. Ecological intensification of cereal production systems: Yield potential, soil quality, and precision agriculture. Proceedings of the National Academy of Sciences of the United States of America, v. 96 , n. 11, p. 5952-5959, 1999. DOI: https://doi.org/10.1073/pnas.96.11.5952
COMMITTEE ON ASSESSING CROP YIELD: SITESPECIFIC FARMING, INFORMATION SYSTEMS, AND RESEARCH OPPORTUNITIES, NATIONAL RESEARCH COUNCIL: BOARD ON AGRICULTURE. Precision Agriculture in the 21st Century: Geospatial and Information Technologies in Crop Management. Washington: National Academy Press., 1997. 168 p.

CONAB. Perspectivas para a agropecuária. v. 7, safra 2019/2020. 2021. Disponível em: $<$ file://C:/Users/crist/Downloads/PerspectivaZparaZaZAgr opecuariaZZ-ZV.7ZZ2019-2020.pdf>. Acesso em 27 out. 2021.

DALCHIAVON, F. C.; CARVALHO, M. P.; NOGUEIRA, D. C.; ROMANO, D.; ABRANTES, F. L. ASSIS, J. T.; OLVEIRA, M. S. Produtividade da soja e resistência mecânica à penetração do solo sob sistema plantio direto no cerrado brasileiro. Pesquisa Agropecuária Tropical, v. 41, n. 1, p.8-19, 2011.

DALLMEYER, A.U.; SCHLOSSER, J.F. Mecanizacion para la agricultura de precisión. In: BLU, R. O.; MOLINA, L. F. Agricultura de precisión - Introducción al manejo sitio específico. Chillán-Chile: INIA, 1999. Cap.3, p.75-104.

FERRAZ, G. A. S.; SILVA, F. M.; ALVES, M. C.; BUENO, R. L.; COSTA, P. A. N. Geostatistical analysis of fruit yield and detachment force in coffee. Precision Agriculture, v. 13, n. 1, p. 76-89, 2012.

FOLEY, J.A.; RAMANKUTTY, N.; BRAUMAN, K.A.; CASSIDY, E.S. Solutions for a cultivated planet. Nature, v.478, n.7369, p.337-342, 2011.

FONSECA, A.A. Agricultura de precisão: conceitos e tecnologias. Revista Pensar Acadêmico, v.3, n.1, 2009. DOI: https://doi.org/10.21576/rpa.2009v5i1.1049

GEBBERS, R.; ADAMCHUK, V. I. Precisionagricultureand food security. Science, v. 327, n. 5967, p. 828-831, 2010.

IGBE. Pesquisa Nacional por Amostra de Domicílios (PNAD). 2015. Disponível em: <https://educa.ibge.gov.br/jovens/conheca-o-

brasil/populacao/18313-populacao-rural-e-urbana.html>. Acesso em 26 out. 2021.

INAMASU, R. Y.; BERNARDI, A. C. C. Agricultura de precisão. In: BERNARDI, A. C. C.; NAIME, J. M.; RESENDE, A. V.; BASSOI, L. H.; INAMASU, R. Y. Agricultura de precisão: resultados de um novo olhar. Brasília, DF: Embrapa, 2014. p. 21-33.

MANTOVANI, E. C. Agricultura de precisão e sua organização no Brasil. In: BORÉM, A. Agricultura de precisão. Viçosa: UFV, 2000. p. 77-108.

MANYIKA, J.; CHUI, M.; MIREMADI, M.; BUGHIN, J.; GEORGE, K.; WILLMOT, P.; DEWHURST, M. A future thatworks: Automation, employment, andproductivity. Mc Kinsey Global Institute, New York. 2017. Disponível em:<mckinsey.com/global-themes/digital-

disruption/harnessing-automationfor-a-future-that-works>. Acesso em: 25out. 2021.

MIRANDA, E. Modelagem e mapas de capacidade de suporte de carga de solos cultivados com cana-de-açúcar. 2006. 97 f. Tese (Doutorado em Agronomia) - Faculdade 
de Ciências Agronômicas, Universidade Estadual Paulista "Júlio de Mesquita Filho", Botucatu, 2006.

MOLIN, J.P. Agricultura de precisão: o gerenciamento da variabilidade. Piracicaba: J.P. MOLIN, 2001. 83 p.

PUSCH, M.; MACHADO, T.P.; AMARAL, L.R. Introdução a Agricultura de Precisão, 03/2019. Disponível em:<www.feagri.unicamp.br/gitap $>$. Acesso em: $08 \mathrm{dez}$. 2021.

RESENDE, A. V.; HURTADO, S. M. C.; VILELA, M. F.; CORAZZA, E. C.; SHIRATSUCHI, L.S. Aplicações da agricultura de precisão em sistemas de produção de grãos no Brasil. In: BERNARDI, A. C. C.; NAIME, J. M.; RESENDE, A. V.; BASSOI, L. H.; INAMASU, R. Y. Agricultura de precisão: resultados de um novo olhar. Brasília, DF: Embrapa, 2014. p. 194-208.

ROZA, D. Novidade no campo: Geotecnologias renovam a agricultura. Revista InfoGEO, n. 11, 2000. Disponível em: <http://www.infogeo.com.br>. Acesso em 08 Dez. 2021.

SANTI, A. L.; AMADO, T. J. C.; EITELWEIN, M. T.; CHERUBIN, M. R.; DA SILVA, R. F.; DA ROS, C. O. Definição de zonas de produtividade em áreas manejadas com agricultura de precisão. Revista Brasileira de Ciências Agrárias, v. 8, n. 3, p. 510-515, 2013. DOI: https://doi.org/10.5039/agraria.v8i3a2489

SILVA, C. B.; VALE, S. M. L. R.; PINTO, F. A. C. ; MÜLLER, C. A. S. ; MOURA, A. D. The economic feasibility of precision agriculture in Mato Grosso do Sul State, Brazil: a case study. Precision Agriculture, v. 8, p. 255-265, 2007. DOI: http://dx.doi.org/10.1007/s11119007-9040-2

SOARES FILHO, R.; CUNHA, J. P. A. R. Agricultura de precisão: particularidades de sua adoção no sudoeste de Goiás - Brasil. Engenharia Agrícola, v.35, n.4, p.689698,2015. DOI: https://doi.org/10.1590/1809-4430Eng.Agric.v35n4p689-698/2015

TOLEDO, J. L. C.; LAROCA, J. V. S.; SILVA, J. P. P.; RIBEIRO, A. S. O uso da geoestatística no preparo do solo: a agricultura de precisão. Revista Científica Eletrônica de Ciências Sociais Aplicadas EDUVALE, p. 68-76, 2020.

VITOUSEK, P.M.; NAYLOR, R.; CREWS, T.; DAVID, M.B. Nutrient Imbalances in Agricultural Development. Science, v.324, n.5934, p.1519-1520, 2009. DOI http://dx.doi.org/10.1126/science. 1170261

OLIVEIRA, A. B., MOURA, C.F.H., GOMES-FILHO, E.; MARCI, C.A., URBAN, L., MIRANDA, M.R. The Impact of Organic Farming on Quality of Tomatoes Is Associated to Increased Oxidative Stress during Fruit Development. PLoS One. Vol. 8, p 1-6, 2013. DOI: http://dx.doi.org/10.1371/journal.pone.0056354

RIBEIRO, M.I.B, FERNANDES, A. J. G., CABO, P.S.A., MATOS, A. M. V. Qualidade nutricional e tecnológica dos alimentos na ótica do consumidor. Revista Mundi Meio Ambiente e Agrárias, v. 2, n. 2, p. 16-1-16-19, 2017. DOI: http://dx.doi.org/10.21575/25254790rmmaa2017vol2n229 $\underline{3}$ 